\title{
Healthcare Encounters Sponsor Defined Identifier
}

National Cancer Institute

\section{Source}

National Cancer Institute. Healthcare Encounters Sponsor Defined Identifier. NCI

Thesaurus. Code C124004.

One or more sponsor defined characters used to identify, name, or characterize the healthcare encounters. 